kidney. The success with which the authors sustain their chosen approach is remarkable. Attempts to interpret biological phenomena in biochemical terms are common enough but they are seldom supported by such a command of general biochemistry. Moreover, some of the difficulty and some of the attraction of liver and kidney regeneration as a subject for research is that it does not lie clearly within the province of any recognized discipline. It was originally opened up by experimental surgeons, but it has since engaged the attention of zoologists, physiologists, biochemists and pathologists. It is a considerable achievement to have organized material from so many diverse sources around a single central theme.

The one weakness in an otherwise excellent book is the brief comparison of regeneration in the two tissues with which it ends. Like everything which has preceded it, this is concise and lucid, but it could with advantage have been longer. The authors are leaders in their respective fields. Many of their experiments have been marked by novelty of approach and extraordinary technical virtuosity. Having devoted 250 pages to describing the existing state of knowledge they might have allowed themselves more than three pages to discuss their conclusions. R. Y. THOMSON

\section{After the Apple}

Gravity and the Organism. Edited by S. A. Gordon and M. J. Cohen. Pp. ix +474. (University of Chicago: Chicago and London, September 1971.) $£ 6.30$.

Although this book covers a diverse and important field of knowledge it will be known to people who work in it as tightly compartmented. Thus, a vertebrate biologist will be familiar with the statoliths of fish and mammals, their mechanical responses to acceleratory forces including gravity and the associated neural mechanisms whereby these responses are translated into appropriate movements or sensation. But this is only part of a much larger picture. His ideas will need to be enlarged; for instance, by reference to the work of plant biologists on the geotropic mechanisms of plants, and in a variety of other directions. For this there is needed a freer diffusion of knowledge between the compartment walls and much is done to achieve this by the compendium of up to date information, well balanced and handsomely presented, which is to be found in this volume. This comprises the texts of thirty-nine papers, together with the discussions relating thereto, which were presented at a symposium on gravity and the organism. at Tuxedo, New York, in 1967. The contents have since been updated to 1971 , the year of publication.

The meeting was sponsored by the Space Science Board of the US National Academy of Science with the support of the National Aeronautics and Space Administration (NASA) and attracted fifty-four participants. Twenty of the papers were from the United States and eighteen from European laboratories, including five from Great Britain. There was also a contribution from Japan. There is an excellent introduction by $\operatorname{Dr} \mathrm{A}$. H. Brown of the University of Pennsylvania, and summaries are provided by Professor O. Lowenstein of Birmingham and Professor A. W. Galston of Yale.

The resulting book is large ; 470 pages are divided into seven parts, covering the reactions to gravity of plants (three parts), invertebrates (one part), and vertebrates (one part). There is also a large part (100 pages) devoted to gravimorphism in plants and animals. The final part contains an interesting summary of recent space studies.

The areas most familiar to me are those concerned with the functional anatomy and gravity sensing mechanisms of the inner ear of animals. These are dealt with by Gualtierotti of Milan and Lowenstein. They are of notable excellence and include an up to date summary of electron microscopic data. Other sections of especial interest are concerned with gravity susception by plants. This, according to some workers, would appear to involve mechanisms essentially similar to those of the animal labyrinth. Here, use is made of statoconia which by virtue of their high specific gravity are displaced by gravity, stimulate the underlying sensory cells and so initiate movements of the animal which are appropriate to its gravitational circumstances. Larsen of Bergen presents very clear evidence that in plants the part of the statoconia is played by certain high density elements which are judiciously categorized as "sedimentary particulates". According to the positioning of the plant with respect to gravity these particulates, in some cases starch granules -amyloplasts-will vary their position

\title{
Human Hair
}

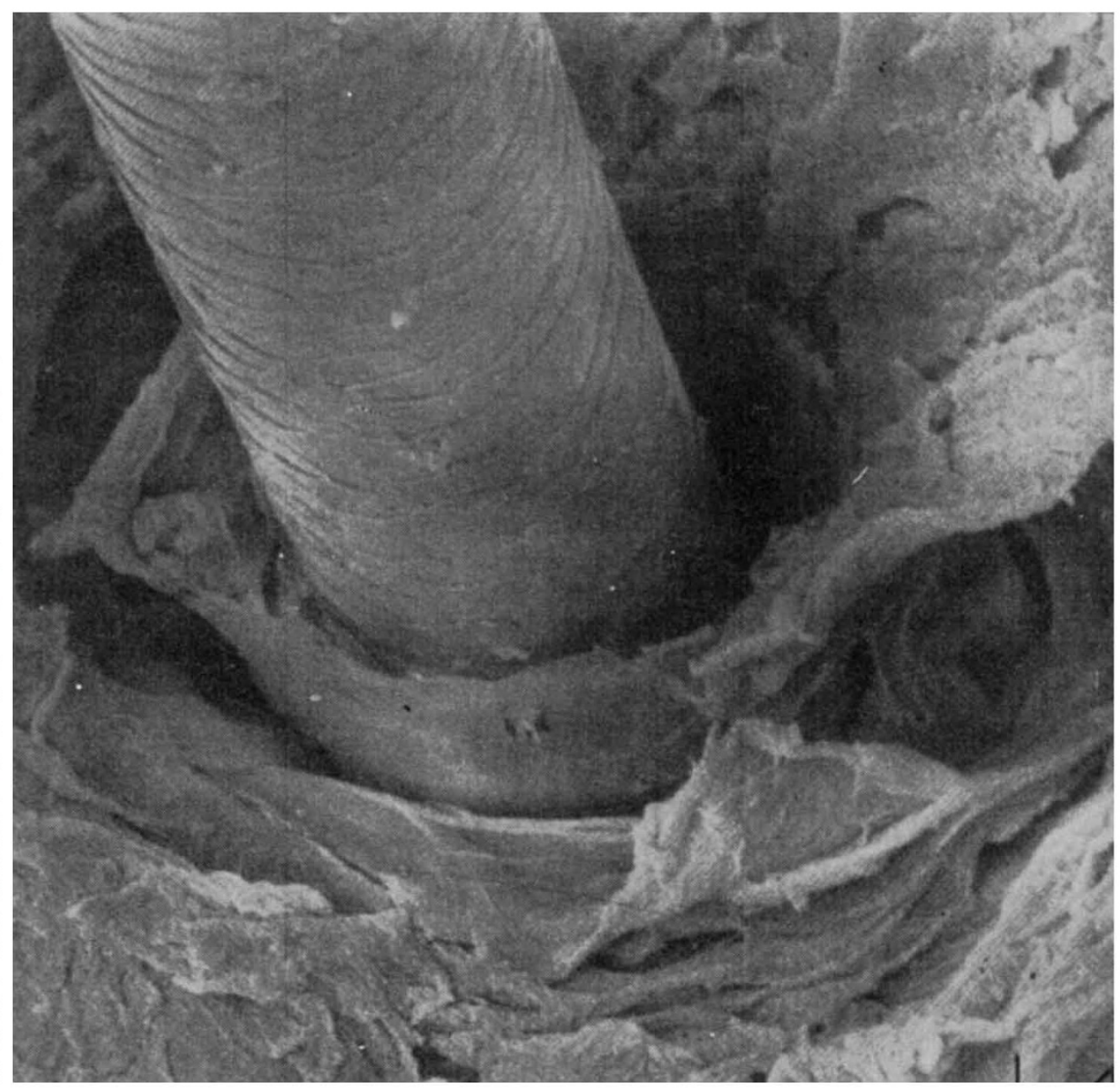

The basal part of a hair of the human head. The epidermal surface is covered with leaflike cells which, when peeled off, will form dandruff. (Magnification $\times 1,020$.) Taken from Atlas of Scanning Electron Microscopy in Medicine, by T. Fujita, J. Tokunaga and $\mathrm{H}$. Inou.

(Tokyo: Igaku Shoin; Amsterdam, London, and New York: Elsevier 1971.) £9.00. 